\title{
Préparation du Canada en cas de grippe pandémique : Stratégie de communication
}

\section{B. Henry ${ }^{1,2}$, au nom du groupe de travail sur le document Préparation du Canada en cas de grippe pandémique (PCGP)*}

\section{Résumé}

En situation d'incertitude et d'imprévisibilité, il est essentiel de communiquer de façon transparente et rapide lors d'une pandémie pour instaurer la confiance et établir la crédibilité des conseils fournis en matière de santé publique. La responsabilité de la communication avec les Canadiens lors d'une pandémie est partagée entre les gouvernements fédéral, provinciaux, territoriaux et locaux. II convient de se doter d'un plan commun pour s'assurer d'une approche cohérente, coordonnée et appropriée en matière de communication. La diversité du Canada sur le plan de sa taille, de sa géographie, des langues que l'on y parle et de sa culture nécessite également l'adoption d'une approche multidimensionnelle de sorte que le message approprié soit communiqué au bon moment, à la bonne personne, dans le format qui convient.

L'annexe traitant des communications et de la liaison avec les intervenants est une stratégie de communication intégrée au document Préparation du Canada en cas de grippe pandémique: Guide de planification pour le secteur de la santé (PCGP) qui a récemment été mise à jour. L'annexe insiste sur l'importance que revêt le fait de communiquer tant avec la population qu'avec les principaux intervenants (p. ex. les fournisseurs de soins de santé, les organisations professionnelles et les décideurs politiques) avant, durant et après une pandémie. Cette stratégie repose sur plusieurs principes directeurs en matière de communications, à savoir privilégier en toute circonstance la santé des Canadiens, fournir de l'information de qualité rapidement, communiquer de façon coordonnée au niveau de l'ensemble des ordres de gouvernement, protéger la confidentialité et, enfin, surveiller la perception du risque de la population et s'y adapter. L'annexe décrit une approche de communication sur les risques, propose des déclencheurs d'intervention pour des pandémies ayant des répercussions variables et inclut un protocole de communication qui sera mis en œuvre à travers le pays, dans l'éventualité d'une pandémie.

\section{Affiliations}

${ }^{1}$ Présidente du groupe de travail sur le PCGP

2 Bureau de l'agent de santé provincial, Victoria (Colombie-Britannique)

*Correspondance : phac.cpip.tg. secretariat-gt.pcp.aspc@canada.ca

Citation proposée : B. Henry au nom du groupe de travail sur le document Préparation du Canada en cas de grippe pandémique (PCGP). Préparation du Canada en cas de grippe pandémique : Stratégie de communication. Relevé des maladies transmissibles au Canada. 2018;44(5):118-22. https://doi.org/10.14745/ ccdr.v44i05a03f

Mots clés : Grippe pandémique, stratégie de communication, perception du risque, communication sur le risque, protocole d'urgence

\section{Introduction}

Lors d'une pandémie, il est impératif de communiquer de façon transparente et rapide pour instaurer, auprès de la population, de la confiance quant à la capacité des responsables de gérer la pandémie et de protéger les Canadiens. À chacune des étapes de la pandémie, le fait de fournir de l'information précise, crédible et récente - en s'assurant que le message approprié soit communiqué au bon moment, par la bonne personne, à l'auditoire qui convient - peut contribuer à protéger la santé de la population, à sauver des vies et à minimiser les perturbations tant sociales qu'économiques.

L'annexe traitant des communications et de la liaison avec les intervenants (1) est une stratégie de communication intégrée au document Préparation du Canada en cas de grippe pandémique: Guide de planification pour le secteur de la santé (2) qui a récemment été mise à jour. L'objet principal de cette annexe tient à la communication fédérale, provinciale et territoriale (FPT) avec la population; on y reconnaît de surcroît l'importance que revêt le fait de communiquer avec les intervenants clés, qu'il s'agisse des fournisseurs de soins de santé, des organisations professionnelles et des décideurs. L'annexe a été mise à jour en tenant compte de l'expérience acquise en marge de la pandémie H1N1 de 2009. Cette pandémie a mis en évidence l'importance d'une communication claire, fréquente et coordonnée avec la population de même qu'avec les intervenants clés et la nécessité d'élaborer des plans adaptés à des pandémies ayant des répercussions variables.

Cette annexe actualisée retient une approche souple et évolutive à l'égard de la planification et inclut des pratiques exemplaires 
au chapitre de la communication sur les risques. Elle intègre une large gamme de méthodes de communication comme le marketing social, les médias sociaux et la consultation des intervenants. On y décrit les rôles et les responsabilités des gouvernements FPT en matière de communication lors d'une pandémie de même que les mécanismes par l'entremise desquels les messages clés sont coordonnés, comme par le truchement de réseaux établis et d'organismes non gouvernementaux. L'annexe est cohérente avec les concepts que l'on retrouve dans le corps du document PCGP; elle encadre les approches en matière de communication en s'appuyant sur les principes directeurs que sont l'éthique, la collaboration, la proportionnalité et la prise de décisions fondées sur des données probantes.

\section{Objectifs}

Lors d'une pandémie, les objectifs en matière de communication sur les risques consistent à informer et à mobiliser les Canadiens, de même que les organisations qui les représentent, quant aux risques que pose la pandémie de sorte que chacun puisse prendre les mesures appropriées. Pour ce faire, on communique I'information pertinente dans les formats qui sont accessibles et adaptés aux besoins de l'auditoire ( $p$. ex. dans des langues différentes et en tenant compte des sensibilités culturelles), cette information étant transmise sur de multiples plateformes et au travers de celles-ci (p. ex. communiquée par des leaders locaux, comme des anciens de la communauté ou des maires de municipalités) et en coordonnant les communications par le biais d'un point de convergence clairement défini ( $p$. ex. l'administrateur en chef de la santé publique). Lors de la pandémie, on fait appel à des réseaux FPT établis afin d'assurer la cohérence du message, de partager des pratiques exemplaires et d'ajuster les approches, au besoin.

Le présent article résume l'annexe traitant des communications et de la liaison avec les intervenants récemment mise à jour du document PCGP (1); il s'inscrit dans le cadre d'une série décrivant l'approche du Canada à l'égard de la préparation en cas de grippe pandémique (3-6).

\section{Contexte canadien}

Le Réseau pancanadien de santé publique (RSP) est le mécanisme par le truchement duquel est coordonnée la réponse en matière de communications lors d'une pandémie. Le RSP est dirigé par un conseil de 17 membres composé de représentants des gouvernements FPT, y compris l'administrateur en chef de la santé publique et des hauts fonctionnaires des gouvernements de toutes les provinces et de tous les territoires responsables de la santé publique. Le RSP inclut un groupe de communications intergouvernementales qui fournit des conseils en matière de communication sur les risques et de marketing social et assure du soutien à cet égard lors d'une pandémie, en facilitant la coordination inter-juridictionnelle et le partage de l'information entre les responsables de la santé publique canadiens.

Du fait de la taille du Canada et de la répartition géographique de sa population, il est vraisemblable qu'une pandémie touche différentes régions à des moments différents et selon des niveaux de gravité variables de sorte que le message transmis doit expliquer la situation qui prévaut dans les contextes global, national et régional, en plus de s'attaquer aux fausses informations qui circulent peut-être.

L'Agence de la santé publique du Canada (ASPC) sert de point de convergence pour les communications nationales sur les questions de santé publique. L'ASPC collabore avec des organismes internationaux (p. ex. l'Organisation mondiale de la santé, le groupe d'action mondial pour la sécurité sanitaire (Global Health Security Action Group) et le groupe de travail trilatéral qui participe au Plan nord-américain contre l'influenza animale et la pandémie d'influenza et assure la coordination avec d'autres ministères fédéraux afin de fournir des conseils en matière de santé aux Canadiens, par exemple sur les précautions à prendre en cas de voyage ou sur la façon de prévenir les maladies et les blessures.

Les provinces/territoires sont la principale source de communications avec la population et les intervenants clés, comme les responsables municipaux et régionaux de la santé ou les organismes ou partenaires des provinces et des territoires, au sein de leurs administrations. L'ASPC et les provinces/territoires coordonnent leurs efforts et leurs approches par le truchement du Groupe des communications du RSP.

L'annexe souligne de surcroît l'importance de faire cadrer les stratégies en matière de communication avec les tendances quant à la façon dont les Canadiens accèdent à l'information en matière de santé et utilisent celle-ci. Les responsables de la planification doivent tenir compte du fait que certains Canadiens n'ont pas d'accès Internet voire disposent d'un accès limité ou peu fiable à ce réseau. II se pourrait par ailleurs qu'il soit plus judicieux de fournir de l'information à certains groupes de population dans des formats et dans des langues différents, par exemple dans le cas des personnes souffrant d'un handicap auditif ou visuel, des personnes dont le niveau d'alphabétisation est faible, des immigrants récents ou des sans-abris. II est possible de retenir plusieurs stratégies pour rejoindre les Canadiens, qu'il s'agisse de la sensibilisation directe des collectivités, des médias traditionnels ( $p$. ex. journaux, téléphone, radio et télévision), d'approches inédites et des nouveaux médias (p. ex. mobilisation des travailleurs d'approche, médias sociaux) en plus d'avoir recours aux médias en ligne.

\section{Principaux éléments de l'approche en matière de communication}

L'annexe traitant des communications et de la liaison avec les intervenants inclut un protocole FPT conjoint applicable aux nouveaux événements touchant la santé publique. Ce protocole désigne six principes directeurs applicables aux communications pancanadiennes qui pourraient et devraient être appliqués dans un contexte de pandémie (voir la boîte de texte).

\section{Perception du risque}

L'annexe souligne l'importance de la perception du risque de la population, laquelle se définit comme « un jugement subjectif que posent les gens quant aux risques et aux avantages associés à un événement ou à des solutions de rechange " [traduction] (1). Lors d'une pandémie, l'incertitude peut être accompagnée d'une forte demande en matière d'information, de sentiments de peur et d'angoisse plus aigus, de la diffusion rapide d'informations inexactes et de spéculations.

La théorie en matière de communication sur les risques laisse entendre que la perception initiale du risque d'une personne est façonnée relativement tôt durant une pandémie, sur la base de l'information disponible, cette perception passant au travers du filtre des croyances personnelles, de l'éducation et des valeurs (7). De surcroît, une fois que ces perceptions ont 
Principes directeurs en matière de communication lors d'une pandémie de grippe

1. Faire passer avant toute chose la santé des Canadiens. Veiller à ce que les Canadiens disposent de l'information dont ils ont besoin pour se protéger et protéger autrui.

2. Fournir de l'information qui s'appuie sur les données probantes les plus récentes. L'information communiquée à la population doit être la plus précise possible puisqu'il pourrait s'avérer essentiel de lutter contre les fausses informations et de permettre à la population de se protéger.

3. Fournir de l'information en temps opportun. La communication rapide peut contribuer à empêcher la propagation des infections, réduisant ainsi la gravité et la durée des épidémies, en plus de permettre de sauver des vies.

4. Communiquer de façon coordonnée. Il importe que tous les gouvernements et les partenaires qui assument conjointement des responsabilités en matière de santé publique harmonisent leurs efforts et veillent à ce que l'information qu'ils fournissent à la population est cohérente et appropriée.

5. Protéger la confidentialité des patients. Le droit du patient à la vie privée doit sous-tendre le message communiqué. À son tour, ce message doit respecter les mesures de protection FPT, qui prévoient un équilibre entre les intérêts en matière de santé publique et les droits de chaque patient.

6. Tenir compte de la perception publique du risque. Le contrôle de la perception publique, des besoins en matière d'information et des préoccupations à cet égard constitue un aspect important de toute intervention en cas de pandémie puisque la perception publique du risque constitue le plus solide indicateur de la volonté de la population à changer de comportement lors d'un événement touchant la santé publique.

été assimilées, elles sont difficiles à altérer. II est donc essentiel de communiquer de façon transparente, ouverte et rapide avec la population et les intervenants quant aux aspects connus et à ceux qui demeurent inconnus (y compris les mesures prises pour mieux cerner la question). L'annexe insiste sur le fait que les responsables de la santé publique et les sources de confiance (p. ex. le personnel infirmier, les médecins, les pharmaciens, les leaders communautaires et les anciens) en plus d'avoir une incidence sur les premiers comportements, établissent également la présence d'une source de conseils et d'avis experts pour aider les Canadiens à mieux comprendre les risques.

Les médias sociaux jouent un rôle de plus en plus important dans la façon dont la population perçoit le risque. Lors d'une pandémie, de nombreux Canadiens chercheront vraisemblablement à obtenir de l'information sur l'épidémie en consultant les médias sociaux (p. ex. Facebook, Twitter). Dans ce contexte, les intervenants peuvent jouer un rôle important sur le plan de la perception publique du risque que pose la pandémie - soit en corrigeant les fausses informations soit en faisant circuler de nouveau des messages précis et cohérents. Les stratégies recommandées en matière de communication tiennent compte de cette nouvelle réalité, tant sur le plan de la façon dont l'information est diffusée que sur celui dont les médias sociaux doivent être surveillés. Il convient de se doter de stratégies pour contrer les risques de désinformation (" fausses nouvelles »). Bien que les médias sociaux ne soient pas à l'origine de fausses nouvelles, ils constituent néanmoins une plateforme largement accessible par le truchement de laquelle des opinions ou de fausses informations peuvent être diffusées de manière convaincante et rapide, comme s'il s'agissait de faits. De l'information crédible doit être présentée rapidement et répétée fréquemment par des sources de confiance.

\section{Adaptabilité}

La pandémie de 2009 a donné lieu à d'importantes variations quant aux activités associées aux vagues de celle-ci en termes de chronologie et d'intensité - à travers le monde comme à travers le Canada et à l'intérieur des provinces et des territoires. Ceci a permis d'établir clairement qu'il convenait de se doter $d^{\prime}$ une approche souple et adaptable en matière de planification. Comme le décrit le document PCGP, l'intervention en cas de pandémie doit être adaptée à la situation locale, des mesures pertinentes étant appliquées au niveau provincial/territorial ou régional/local, selon ce qu'exige la situation.

Le document PCGP met de l'avant quatre scénarios de planification qui décrivent les répercussions possibles $d^{\prime}$ 'une pandémie, qui varient d'un niveau faible à élevé, compte tenu de la transmissibilité du virus et du niveau de gravité clinique. Le tableau 1 présente ces scénarios de planification et de répercussions au chapitre de la communication sur les risques.

\section{Évaluation permanente}

Il est nécessaire d'assurer une évaluation systématique de I'intervention globale en matière de communication à trois étapes clés de la pandémie :

- Continuellement : Pour permettre aux administrations d'adapter leurs plans en tenant compte de l'évolution de la situation et de partager, avec leurs homologues FPT, les connaissances acquises afin de s'assurer de mettre de l'avant une réponse transparente et complémentaire, à travers le Canada, durant l'épidémie.

- Entre la première et la deuxième vagues : Compte tenu du fait que les pandémies précédentes qui ont frappé l'Amérique du Nord ont connu deux vagues successives, lors de la " pause " qui survient entre celles-ci, les administrations devraient faire le point sur leurs interventions individuelles et collectives en marge de la première vague de l'épidémie et ajuster leurs activités en conséquence, avant que ne survienne une éventuelle deuxième vague.

- Après la pandémie : Les évaluations varient d'une administration à l'autre, selon l'impact de la pandémie de même que la portée et l'échelle de l'intervention en matière de communication. Les administrations sont incitées à évaluer l'efficacité de leurs documents de communication avec leurs homologues FPT.

Pour contribuer à ces efforts, I'annexe comprend une liste $d^{\prime}$ indicateurs de rendement liés tant aux processus qu'aux résultats en matière de communication qui peuvent être appliqués afin d'évaluer l'efficacité des approches et des outils de communication. 
Tableau 1 : Répercussions et adaptations recommandées à l'intervention en matière de communication pour des pandémies ayant des répercussions variables

\begin{tabular}{|c|c|c|}
\hline \multirow{2}{*}{$\begin{array}{c}\text { Trans- } \\
\text { missibilité }\end{array}$} & \multicolumn{2}{|c|}{ Gravité clinique } \\
\hline & FAIBLE & ÉLEVÉ \\
\hline ÉLEVÉ & $\begin{array}{l}\text { Scénario B (répercussions } \\
\text { modérées) : Virus } \\
\text { grippal présentant une } \\
\text { transmissibilité élevée et une } \\
\text { virulence faible } \\
\text { - Prévoir que la } \\
\text { transmissibilité } \\
\text { élevée rehaussera le } \\
\text { niveau d'inquiétude } \\
\text { de la population et } \\
\text { augmentera la demande } \\
\text { d'antiviraux ou de vaccins } \\
\text { pandémiques } \\
\text { Élaborer une approche } \\
\text { de communications afin } \\
\text { de renforcer les mesures } \\
\text { de santé publique } \\
\text { (vaccination, hygiène des } \\
\text { mains) et la nécessité } \\
\text { de prendre soin des } \\
\text { personnes malades } \\
\text { lncorporer des messages } \\
\text { portant sur le bien-être } \\
\text { en milieu de travail aux } \\
\text { communications internes } \\
\text { (p. ex. dans les bulletins } \\
\text { destinés aux employés) } \\
\text { Mener des campagnes } \\
\text { de marketing afin } \\
\text { d'inciter chacun à } \\
\text { adopter de bonnes } \\
\text { pratiques en matière de } \\
\text { santé, à rester chez soi } \\
\text { lorsque l'on est malade, } \\
\text { etc. } \\
\text { Prévoir les questions } \\
\text { et les préoccupations } \\
\text { des médias et de la } \\
\text { population sur les } \\
\text { suggestions relatives à la } \\
\text { vaccination }\end{array}$ & $\begin{array}{l}\text { Scénario D (répercussions } \\
\text { élevées) : Virus } \\
\text { grippal présentant une } \\
\text { transmissibilité élevée et } \\
\text { une virulence élevée } \\
\text { - Prévoir que les } \\
\text { questions relatives } \\
\text { à la vaccination } \\
\text { (p. ex. disponibilité } \\
\text { des vaccins, accès } \\
\text { prioritaire, sécurité et } \\
\text { efficacité) domineront } \\
\text { les communications } \\
\text { avec la population } \\
\text { Surveiller et expliquer } \\
\text { de manière proactive } \\
\text { les différences quant } \\
\text { aux mesures ou aux } \\
\text { recommandations } \\
\text { en matière de santé } \\
\text { publique relativement à } \\
\text { 'utilisation des vaccins } \\
\text { et des antiviraux } \\
\text { (p. ex. entre différentes } \\
\text { provinces ou différents } \\
\text { territoires, entre le } \\
\text { Çanada et les } \\
\text { Ettats-Unis). } \\
\text { S'assurer de } \\
\text { dénombrer les cas } \\
\text { de façon cohérente, } \\
\text { en instaurant une } \\
\text { coordination entre les } \\
\text { administrations }\end{array}$ \\
\hline FAIBLE & $\begin{array}{l}\text { Scénario A (répercussions } \\
\text { faibles) : Virus grippal } \\
\text { présentant une } \\
\text { transmissibilité et une } \\
\text { virulence faibles } \\
\text { - Prévoir une certaine } \\
\text { complaisance de la part } \\
\text { de la population } \\
\text { (c.-à-d. que les gens } \\
\text { pourront estimer qu'ils } \\
\text { ne sont eux-mêmes pas } \\
\text { à risque) } \\
\text { Prévoir un niveau } \\
\text { de communication } \\
\text { approprié pour éviter } \\
\text { toute saturation de } \\
\text { l'information } \\
\text { - Prévoir que la perception } \\
\text { du risque de la } \\
\text { population pourrait } \\
\text { s'axer principalement sur } \\
\text { le caractère approprié } \\
\text { des efforts en matière } \\
\text { d'intervention } \\
\text { - Être préparé à des } \\
\text { changements rapides } \\
\text { quant à la perception du } \\
\text { risque de la population } \\
\text { (p. ex. suite a un décès) }\end{array}$ & $\begin{array}{l}\text { Scénario C (répercussions } \\
\text { modérées) : Virus } \\
\text { grippal présentant une } \\
\text { transmissibilité faible et } \\
\text { une virulence élevée } \\
\text { - Prévoir que la virulence } \\
\text { élevée (dans le cas } \\
\text { d'un virus causant une } \\
\text { maladie clinique grave) } \\
\text { rehaussera le niveau } \\
\text { de préoccupation de la } \\
\text { population } \\
\text { - ntervenir de façon } \\
\text { proactive à l'égard } \\
\text { des préoccupations } \\
\text { en communiquant } \\
\text { régulièrement par } \\
\text { le truchement de } \\
\text { multiples formes de } \\
\text { médias } \\
\text { Cibler les } \\
\text { communications vers } \\
\text { les groupes à risque } \\
\text { élevé }\end{array}$ \\
\hline
\end{tabular}

\section{Intégration des stratégies de communication avec d'autres composantes du document PCGP}

La formulation d'un message efficace et le recours à une approche en matière de communication sur les risques constituent des aspects fondamentaux de tout élément d'une intervention en cas de pandémie. À titre d'exemples de la façon dont ces principes s'appliquent à d'autres volets du document PCGP, figurent la communication des décisions concernant la distribution initiale des vaccins, l'établissement de l'ordre des priorités des vaccins dans l'éventualité d'une pénurie d'approvisionnement ou les messages touchant les mesures de santé publique comme sur le plan de la nécessité d'adopter des mesures d'éloignement social ou d'auto-isolement, lorsque l'on est frappé par la maladie.

\section{Besoins en matière de recherche}

La recherche concernant la communication sur les risques, la gestion des intervenants, les sciences du comportement, la modélisation et le suivi peuvent jouer un rôle clé au niveau de la préparation et de l'intervention en cas de pandémie. Il est absolument indispensable de se pencher sur de nouveaux outils, techniques et méthodologies de communication. Bien qu'une partie importante de ces travaux de recherche peuvent être effectués durant la période qui survient entre deux pandémies, une partie de ceux-ci ne peut être réalisée que lors d'une pandémie (p. ex. s'agissant de comparer les taux d'adoption des vaccins en fonction des stratégies de communication de diverses administrations). Comme il se peut que l'intervalle séparant deux événements soit long, il convient de se doter de stratégies proactives pour tirer parti de ces possibilités d'apprentissage peu fréquentes mais néanmoins inestimables et les mettre à profit.

\section{Discussion}

La survenue d'une épidémie donne lieu à énormément d'incertitudes, ce qui se traduit par une demande élevée en matière d'information, de sorte que chacun puisse prendre de bonnes décisions. II est essentiel de communiquer de façon transparente, fréquemment et tôt dans le processus avec la population et les intervenants quant aux aspects qui sont connus et à ceux qui demeurent inconnus pour réduire les sentiments de peur et d'angoisse tout en répliquant aux fausses informations et aux spéculations - ce qui demeure un défi compte tenu du fait que l'information circule rapidement, par les médias électroniques.

Le Canada présente un profil diversifié sur le plan de sa taille, de sa géographie, de sa culture, de ses langues et des besoins de sa population. Il importe de se doter de communications spécialisées ou adaptées pour adopter une approche inclusive et tenir compte des façons traditionnelles et de rechange par le truchement desquelles les Canadiens ont accès à l'information, tout en prenant acte des nouvelles technologies et des tendances quant à la façon dont les Canadiens sont informés. Comme cette activité est à forte intensité de main-d'œuvre et que ces messages peuvent être délicats à préparer lors d'une pandémie, ils doivent être élaborés d'avance. L'adoption d'une approche marquée au sceau de la collaboration à l'égard de l'élaboration, de la mise à l'épreuve et de l'évaluation des stratégies applicables au message contribuerait à renforcer l'approche optimale à retenir lors d'une pandémie.

Le document PCGP, auquel est joint l'annexe traitant des communications et de la liaison avec les intervenants, vise à fournir aux gouvernements FPT des conseils en matière de planification pour faire face aux défis au chapitre des communications et des conseils de planification lors d'une grippe pandémique. L'annexe est un document qui ne cessera d'évoluer et comme c'est le cas du corps principal du document PCGP et 
des autres annexes techniques, il sera revu et mis à jour tous les cinq ans.

\section{Conclusion}

Le caractère urgent et imprévisible des pandémies nécessite que l'on se dote d'une approche systématique en matière de communication sur les risques. Au début d'une pandémie, il se peut que l'on ne dispose que de peu de données probantes sur les répercussions de celle-ci et sur les populations les plus vulnérables. L'annexe traitant des communications et de la liaison avec les intervenants a été conçue de manière à mettre de l'avant des stratégies de communication adaptables pour les diverses activités associées aux vagues pandémiques d'une façon qui permettra d'accroître la confiance et de favoriser I'autonomisation des Canadiens.

\section{Déclarations de l'auteur}

Membres du groupe de travail sur le document Préparation du Canada en cas de grippe pandémique (GTPCGP) : B Henry (présidente), C Alfieri, S Gantt, I Gemmill, T Hatchette, E Henry, B Schwartz

PCGP Secrétariat : L Paddle, R Stirling, C Comeau

ASPC : P Salsbury, J Williams, S Smith, S Rempel

\section{Conflit d'intérêt}

Aucun.

\section{Remerciement}

L'auteur souhaite remercier Michel Amar qui a préparé au première ébauche du présent résumé.

\section{Financement}

Les travaux du Groupe de travail sur le document Préparation du Canada en cas de grippe pandémique sont appuyés par l'Agence de la santé publique du Canada.

\section{Références}

1. Réseau pancanadien de santé publique. Préparation du Canada en cas de grippe pandémique : Guide de planification pour le secteur de la santé. Ottawa (ON): Agence de la santé publique du Canada; 2018. Annexes Communications. https:// www.canada.ca/fr/sante-publique/services/grippe-influenza/ preparation-canada-cas-grippe-pandemique-guid e-planification-secteur-sante/annexe-traitant-communicatio ns-liason-avec-intervenants.html

2. Réseau pancanadien de santé publique. Préparation du Canada en cas de grippe pandémique : Guide de planification pour le secteur de la santé. Ottawa (ON): Agence de la santé publique du Canada; 2015 https://www.canada.ca/fr/santepublique/services/grippe-influenza/preparation-canada-casgrippe-pandemique-guide-planification-secteur-sante.html

3. Henry B au nom du Groupe de travail sur la préparation du Canada en cas de grippe pandémique (GTPCGP). Préparation du Canada en cas de grippe pandémique : Guide de planification pour le secteur de la santé. Relevé des maladies transmissibles au Canada. 2018;44(1):7-10. http://dx.doi. org/10.14745/ccdr.v44i01a02

4. Henry B au nom du Groupe de travail sur la préparation du Canada en cas de grippe pandémique (GTPCP). Préparation du Canada en cas de grippe pandémique : Stratégie de laboratoire. Relevé des maladies transmissibles au Canada. 2018;44(1):11-5. http://dx.doi.org/10.14745/ccdr.v44i01a03

5. Henry B au nom du Groupe de travail sur la préparation du Canada en cas de grippe pandémique (GTPCP). Préparation du Canada en cas de grippe pandémique : Stratégie de surveillance. Relevé des maladies transmissibles au Canada. 2018;44(1):16-20. http://dx.doi.org/10.14745/ccdr.v44i01a04

6. Henry B, Gadient S au nom du Groupe de travail sur la préparation du Canada en cas de grippe pandémique. Stratégie canadienne de vaccination en cas de pandémie. Relevé des maladies transmissibles au Canada. 2017;43(7/8):185-9. http://dx.doi.org/10.14745/ccdr.v43i78a05

7. Santé Canada. Cadre de communication stratégique des risques dans le contexte de la gestion intégrée des risques à Santé Canada et à l'Agence de santé publique du Canada. Ottawa (ON) : Santé Canada; 2006. https://www.canada.ca/fr/ sante-canada/organisation/a-propos-sante-canada/rapportspublications/cadre-communication-strategique-risques-santecanada-agence-sante-publique-canada.html 